\title{
Sulla trasformazione delle curve di sondaggio di resistività differenziale trasversa in curve di Schlumberger
}

\author{
(On the transformation of transverse differential resistivity \\ to Schlumberger sounding curves)
}

D. Patella $(*)-$ D. Schitone $(* *)$

Ricevuto il 22 Dicembre, 1973

\begin{abstract}
Riassunto. - Si descrive un metodo per trasformare una curva di sondaggio differenziale trasverso, ottenuta con un dispositivo elettrodico differenziale, nella curva Schlumberger relativa alla stessa situazione stratigrafica. Partendo dall espressione generale che lega la resistività apparente differenziale a quella Sclılumberger, è possibile, con aleune approssimazioni, derivare un facile procedimento numerico per poter compiere la trasformazione. Vengono inoltre fatte alcune considerazioni sull'applicabilita del metodo.
\end{abstract}

Summar. - A method is described to transform a transverse differential sounding curve to the form of a Schlumberger sounding curve. Starting from the general expression which relates the transverse differential apparent resistivity to the Schlumberger apparent resistivity, it is possible, with some approximations, to derive an easy numerical procedure in order to carry out the transformation. Some considerations are given about the applicability of the method.

\section{1. - INTRODUZIONE}

Il dispositivo differenziale può essere efficacemente adoperato quando sono richiesti, in problemi di prospezione geoelettrica, un maggiore potere risolutivo ed una maggiore profondità d'indagine rispetto ai convenzionali dispositivi elettrodici.

(*) Osservatorio di Geofisica e Fisica Cosmica - Universita di Bari. (**) Istituto di Geodesia e Geofisiea - Universita di Bari. 
A questo scopo sono stati proposti due metodi di sondaggio differenziale:

a) il sondaggio differenziale longitudinale (LDS - longitudinal differential sounding) $\left({ }^{3}\right)$;

b) il sondaggio differenziale trasverso (TDS - transverse differential sounding) ( $\left.{ }^{4}\right)$.

Il dispositivo elettrodico in entrambi i metorli è lo stesso (fig. 1), ma è differente la tecnica di misura per ottenere le corrispondenti resistività apparenti.

Le relazioni che definiscono le resistività apparenti longitudinale $\bar{Q}_{I, 4}$ e trasversa $\bar{o}_{t / 1}$ sono date da (4)

$$
\begin{gathered}
\bar{\varrho}_{L \Delta}=\frac{\mathrm{d} L}{\mathrm{~d}\left(L / \bar{\varrho}_{s}\right)}-\frac{\bar{\varrho}_{s}^{2}}{\bar{\varrho}_{s}-L \partial \bar{\varrho}_{s} / \partial L} \\
\bar{\varrho}_{t \Delta}=\frac{\mathrm{d}\left(\bar{\varrho}_{s} L\right)}{\mathrm{d} L}=\bar{\varrho}_{s}+L \partial \bar{\varrho}_{s} / \partial L
\end{gathered}
$$

dove $\bar{\varrho}_{s}$ è la resistività apparente Schlumberger ed $L$ la semidistanza tra gli elettrorli di corrente nel dispositivo di Schlumberger.

I valori di $\bar{Q}_{L \Delta}$ e $\bar{\varrho}_{t \perp}$ possono essere espressi in quantità che sono facilmente misurabili in campagna usando l'espressione generale che definisce $\varrho_{s}$. Dalle [1.1] e [1.2] si ottiene (v. Appendice)

$$
\begin{aligned}
& \bar{\varrho}_{L A} \simeq \frac{K_{L}}{I_{1}} \frac{\Delta V_{1} \Delta V_{2}}{\Delta V_{1}-\Delta V_{2}} \\
& \bar{\varrho}_{t \Delta} \simeq \frac{K_{t}}{I_{1}}\left(\Delta V_{2}-\Delta V_{1}\right)
\end{aligned}
$$

essendo $K_{L}$ e $K_{t}$ i fattori geometrici rispettivamente per l'LDS ed il TDS; $\Delta V_{1}$ la d.d.p. generata dalla corrente $I_{1}$ che fluisce tra gli elettrodi interni $A$ e $B ; \Delta V_{2}$ quella generata dalla corrente $I_{2}$ tra gli elettrodi esterni $\Lambda^{\prime}$ e $B^{\prime}$ (v. fig. 1).

Dall'analisi della [1.3] risulta evidente che per valutare $\bar{\varrho}_{I \Delta}$ e necessario misurare separatamente $\Delta V_{1}$ e $\Delta V_{2}$, ottenendo così anche due valori di $\bar{\rho}_{s}$ relativi alle disposizioni $A M N B$ e $B^{\prime} M N \Lambda^{\prime}$. In pratica, quindi, quandlo si efrettua un sondaggio $\operatorname{LDR}\left({ }^{*}\right)$, automaticamente si porta a termine un sondaggio Schlumberger.

(*) LDR - longitudinal differential resistivity.

TDR - transverse differential resistivity. 
Per valutare invece $\bar{o}_{i \dot{ }}$ dalla [1.4] è sufficiente la sola misura di $\Delta(\Delta V)=\Delta V_{2}-\Delta V_{1}$ che può essere effettuata direttamente mediante l'invio simultaneo nel terreno delle correnti $I_{1}$ e $I_{2}$. Con questo modo di procedere si riducono gli errori introdotti dalla misura separata di $\Delta V_{1}$ e $\Delta V_{2}$ e si ottiene inoltre un notevole risparmio di tempo nelle operazioni di campagna.

Pertanto un sondaggio TDR non è necessariamente accompagnato da un sondaggio Schlumberger.

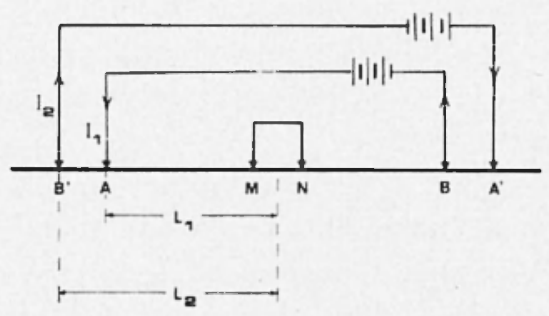

Fig. 1 - Dispositivo elettrodico differenziale.

\section{2. - INTERPRETAZIONE QUANTITATIVA DEI SONDAGGI DIFFERENZIALI}

L'interpretazione quantitativa dei sondaggi LDR e TDR è stata sinora condotta col metodo usuale di confronto con curve teoriche. Per la costruzione di queste ultime sono utilizzabili tre metodi (4):

a) calcolo delle curve teoriche sulla base delle espressioni matematiche che forniscono $\bar{\varrho}_{L,}$ e $\bar{\varrho}_{t \Delta}$ in funzione di $L$. Questo metodo è il più naturale ma, come è noto, richiede una gran mole di lavoro e l'uso del calcolatore elettronico;

b) calcolo delle curve teoriche LDR e TDR trasformando in esse quelle di Schlumberger sulla base delle relazioni [1.1] e [1.2]. Tale trasformazione è possibile solo per quelle situazioni per le quali le curve Schlumberger sono state calcolate;

c) determinazione di $\varrho_{L \Delta}$ e $\bar{\varrho}_{t A}$ combinando opportunamente i valori di $\bar{\varrho}_{s}, \bar{\varrho}_{r}, \bar{\varrho}_{y}$ e $\bar{\varrho}_{x}$, essendo $\varrho_{r}, \bar{\varrho}_{y}$ e $\bar{\varrho}_{x}$ le resistività apparenti per il dispositivo dipolare radiale, perpendicolare e parallelo. $A$ parte la scarsa praticità, quest'ultimo metodo è in realtà possibile solo per un numero molto limitato di modelli stratigrafici per i quali sono disponibili le curve teoriche dipolari. 
Da quanto detto segue che sarebbe di grande interesse disporre di un metodo che permetta di trasformare una curva di resistività differenziale nella forma che si sarebbe ottenuta con il dispositivo di Schlumberger, senza introdurre variazioni alla normale tecnica di campagna. Il vantaggio derivante da una tale trasformazione è notevole: in tal modo sarebbe possibile in più utilizzare direttamente tutti i metodi attualmente esistenti per l'interpretazione delle curve di Schlumberger.

Questo problema non si pone nel caso di sondaggi LDR. Infatti poiché, come si è detto, si ottiene necessariamente la corrispondente curva Schlumberger, è sufficiente interpretare quantitativamente quest'ultima sulla base delle informazioni qualitative che si deducono dall'analisi della curva LDR.

La nostra attenzione è perciò rivolta solo alla possibile trasformazione della curva TDR nella corrispondente curva Schlumberger.

Il metodo che descriviamo presenta analogia con quello già usato per la trasformazione delle curve dipolari in Schlumberger $\left(^{2}\right)$.

\section{3. - Teoria della trasformazione delle curve tDR in curve SCHLUMBERGER}

Riconsideriamo la relazione [1.2]

$$
\bar{\varrho}_{t \Lambda}=\bar{\varrho}_{s}+L \partial \bar{\varrho}_{s} / \partial L \text {. }
$$

Questa puó essere trattata come un'equazione diflerenziale lineare del prim'ordine la cui soluzione generale è data da

$$
\bar{\varrho}_{\delta}=e^{-\int d L / L}\left(\int \bar{\varrho}_{t \Lambda} e^{\int d L / L} d L / L+c\right)
$$

Affinché sia sempre soddisfatta la condizione

$$
\lim _{L \rightarrow 0} \bar{\varrho}_{s} \neq \infty
$$

la costante $c$ deve essere identicamente nulla. Pertanto la [3.1] diventa

$$
\bar{\varrho}_{s}=\frac{1}{L} \int \bar{\varrho}_{t \Delta} \mathrm{d} L .
$$

In pratica, affinché si possa eseguire facilmente l'integrazione al secondo membro della [3.2], procediamo come segue: 
a) dividiamo l'intero intervallo $0 \leqslant L \leqslant \infty$ in una serie di intervalli parziali contigui, di ampiezze in generale differenti tra di loro, del tipo

$$
l_{j} \leqslant L \leqslant l_{j+1}
$$

con $j=1,2,3, \ldots \ldots, n-1$;

b) interpoliamo in ogni intervallo parziale la funzione $\bar{\varrho}_{t_{\Delta}}(L)$ con l'espressione algebrica

$$
\bar{\varrho}_{t \Delta}(L)=a_{j} L^{b_{j}}
$$

che, in scala logaritmica, rappresenta l'equazione di una retta.

Imponiamo ora la seguente condizione: in ogni punto $l_{j}$ sull'asse positivo $L$ il valore $\varrho_{+}\left(l_{j}\right)$ sia noto. Tale condizione è, in campagna, ovviamente soddisfatta qualunque sia il numero di punti $l_{j}$, poiché $\bar{\varrho}_{t \Delta}\left(l_{j}\right)$ è la resistività apparente misurata ad ogni predeterminata spaziatura $l_{j}$.

Nessuna condizione restrittiva viene invece imposta sulla scelta degli intervalli parziali del tipo [3.3]; è, comunque, consigliabile sceglierli i più piccoli possibili, compatibilmente con le difficoltà di campagna, in modo da migliorare l'approssimazione data dalla [3.4].

Ponendo la [3.4] nella [3.2] ed integrando, si ottiene in ogni intervallo parziale

$$
\bar{\varrho}_{s}=\left(\frac{1}{1+b_{j}}\right) a_{j} L^{b_{j}}+a_{j} k / L
$$

dove $k$ è una nuova costante derivante dall'integrazione e necessaria a soddisfare la condizione che in ogni punto comune a due intervalli parziali contigui $\bar{\varrho}_{s}$ deve essere continua.

Se poniamo

si ottiene

$$
c_{j}=a_{j} k
$$

$$
\vec{\varrho}_{s}=\left(\frac{1}{1+b_{j}}\right) a_{j} L^{b_{j}}+c_{j} / L
$$

che più concisamente può essere riscritta

$$
\bar{\varrho}_{s}=\left(\frac{1}{1+b_{j}}\right) \bar{\varrho}_{\Delta t}+c_{J} / L
$$

valida per $l_{j} \leqslant L \leqslant l_{j+1}$ e con $j=1,2, \ldots, n-1$.

Nel punto $l_{j}$ si ha

$$
\bar{\varrho}_{s}\left(l_{j}\right)=\left(\frac{1}{1+b_{j}}\right) \bar{\varrho}_{t \Delta}\left(l_{j}\right)+c_{j} / l_{j}
$$


Nella [3.9] $l_{j}$ e $\bar{\varrho}_{t / 1}\left(l_{3}\right)$ sono entrambe quantità note; per ottenere, quindi, il valore di $\varrho_{s}\left(l_{j}\right)$ basta determinare $b_{j}$ e $c_{j}$.

Riferendoci a $b_{j}$, $\grave{e}$ sufficiente considerare che, dalle ipotesi di partenza, risulta

$$
\left\{\begin{array}{l}
\log \bar{o}_{l_{A}}\left(l_{j}\right)=\log a_{j}+b_{j} \log l_{j} \\
\log \bar{o}_{t \Delta}\left(l_{j+1}\right)=\log a_{j}+b_{j} \log l_{j+1}
\end{array}\right.
$$

Risolvendo il sistema [3.10] nelle incognite $\log a_{j}$ e $b_{j}$, si ottiene

$$
b_{j}=\frac{\log \frac{\bar{o}_{t .1}\left(l_{j+1}\right)}{\bar{\varrho}_{t \Delta}\left(l_{j}\right)}}{\log \frac{l_{j+1}}{l_{j}}}
$$

Riferendoci ora a $c_{j}$, procediamo come segue: consideriamo l'intervallo $l_{j-1} \leqslant L \leqslant l_{j}$, contiguo e precerlente $l_{j} \leqslant L \leqslant l_{j+1}$, dove è

$$
\bar{\varrho}_{s}=\left(\frac{1}{1+b_{j-1}}\right) u_{j-1} L^{b_{j-1}}+c_{j-1} / L
$$

Per la condizione di continuità di $\bar{g}_{s}$ in $l_{j}$ segue che

$$
\left(-\frac{1}{1+b_{j}}\right) a_{j} l_{j}^{b_{j}}+c_{j} / l_{j}=\left(\frac{1}{1+b_{j-1}}\right) a_{j-1} l_{j}^{b_{j-1}}+c_{j-1} / l_{j}
$$

Da quest'ultina si ottiene la seguente formula di ricorrenza

$$
c_{j}=c_{j-1}-\gamma_{j}+\beta_{j-1}
$$

dove

$$
\begin{aligned}
& \gamma_{j}=\left(\frac{1}{1+b_{j}}\right) m_{3 i_{j} i_{j}}=\left(\frac{1}{1+b_{j}}\right) \underline{Q}_{l_{3}}\left(l_{j}\right) l_{j} \\
& \beta_{j-1}=\left(\frac{1}{1+b_{j-1}}\right) a_{j-1} l_{j}^{1+b_{j-1}}=\left(\frac{1}{1+b_{j-1}}\right) \bar{\varrho}_{t \Delta}\left(l_{j}\right) l_{j}
\end{aligned}
$$

Mediante la [3.14] è possibile ottenere $c_{j}$ conoscendo $e_{j-1}$ e cosi via di seguito, essendo $\beta_{j-1}$ e $\gamma_{j}$ quantità note per ogni $j$. In pratica ̀̀ sufficiente trovare il primo valore $c_{0}$ per ottenere tutti i successivi $c_{\text {s }}$.

4. - DETERMINAZIONE DELLE COSTANTI $C_{3}$

Poiché ì

$$
\lim _{L \rightarrow 0} \varrho_{: A}(L)=Q_{1}
$$


con $\varrho_{1}$ la resistività del terreno superficiale, è sempre possibile trovare un punto $l_{1}$, sufficientemente piccolo, per cui si possa ritenere

$$
\bar{\varrho}_{t A}\left(l_{1}\right)=\varrho_{1} \text {. }
$$

Ne consegue che

$$
\bar{\varrho}_{t \Delta}(L) \simeq \varrho_{1}
$$

nell'intero intervallo $0 \leqslant L \leqslant l_{1}$.

Ponendo la [4.3] nella [3.2] ed integrando, si ha

$$
\bar{\varrho}_{s}(L)=\varrho_{1}+c_{0} / L
$$

per $0 \leqslant L \leqslant l_{1}$.

Dalla [4.4] segue che

$$
\lim _{L \rightarrow 0} \bar{\varrho}_{s}(L)=\varrho_{1}
$$

se e solo se

$$
c_{\mathrm{o}}=0
$$

Perciò, partendo dalla [4.6] è possibile ottenere tutti i successivi $c_{j}$ usando la formula di ricorrenza [3.14].

In pratica, per ottenere una curva di campagna di $\stackrel{o}{i<}_{i}$ trasformabile nell'equivalente in $\vec{\varrho}_{s}$, ̀̀ necessario determinare direttamente in campagna l'andamento asintotico a sinistra della curva, o, quando ciò è difficoltoso, eseguire un'estrapolazione a sinistra della curva che rappresenti il più probabile andamento asintotico.

\section{5. - ESEMPI TEORICI DI TRASFORMAZIONE}

Mostriamo ora alcuni esempi di trasformazioni eseguite su curve teoriche di $\bar{D}_{i \Delta}$ riportate da Zohdy $\left({ }^{4}\right)$ e tutte riferite a modelli di terreni a tre strati orizzontali e lateralmente omogenei.

\section{a) Sezione geoclettrica di tipo $H$}

La fig. 2 riporta la trasformazione della curva teorica di $\overline{\bar{o}}_{i\llcorner}$ per una sezione di tipo $H$ con i seguenti parametri

$$
\varrho_{1}=1, \varrho_{2}=0.4, \varrho_{3}=1 ; h_{1}=1, h_{2}=1 \tilde{5} .
$$


TABelLa I $(*)$

\begin{tabular}{|c|c|c|c|c|c|c|}
\hline l) & $\bar{e}_{t, j}\left(l_{j}\right)$ & $b_{j}$ & $\beta_{j}$ & $\gamma_{j}$ & $c_{j}$ & $\bar{\varrho}_{s}\left(l_{j}\right)$ \\
\hline 0.3 & 1.000 & 0.0000 & 0.4000 & 0.3000 & 0.0000 & 1.000 \\
\hline 0.4 & 1.000 & -0.2299 & 0.6168 & 0.5194 & -0.1194 & 1.000 \\
\hline 0.5 & 0.950 & -0.2965 & 0.7676 & 0.6752 & -0.1778 & 0.995 \\
\hline 0.6 & 0.900 & -0.2815 & 0.9241 & 0.7516 & -0.1618 & 0.983 \\
\hline 0.8 & 0.830 & -0.5144 & 1.5239 & 1.3674 & -0.6051 & 0.953 \\
\hline 1 & 0.740 & -0.8827 & 6.4450 & 6.3086 & -5.3898 & 0.919 \\
\hline 1.2 & 0.630 & -1.2477 & -2.8421 & -3.0521 & 4.1073 & 0.879 \\
\hline 1.6 & 0.440 & -1.3576 & -1.8177 & -1.9687 & 3.2339 & 0.791 \\
\hline 2 & 0.325 & -1.0870 & -7.3270 & -7.4713 & 8.8875 & 0.708 \\
\hline 2.5 & 0.255 & -0.3325 & 1.0787 & 0.9551 & 0.6048 & 0.624 \\
\hline 3 & 0.240 & 0.4478 & 0.7542 & 0.4973 & 1.1862 & 0.561 \\
\hline 4 & 0.273 & 0.5696 & 0.9875 & 0.6957 & 1.2447 & 0.500 \\
\hline 5 & 0.310 & 0.5867 & 1.3046 & 0.9769 & 1.2553 & 0.446 \\
\hline 6 & 0.345 & 0.4262 & 2.1876 & 1.4514 & 1.1085 & 0.427 \\
\hline 8 & 0.390 & 0.4376 & 2.9911 & 2.1703 & 1.1258 & 0.412 \\
\hline 10 & 0.430 & 0.3699 & 4.0295 & 3.1389 & 0.9780 & 0.412 \\
\hline 12 & 0.460 & 0.4262 & 5.8337 & 3.8704 & 1.1371 & 0.417 \\
\hline 16 & 0.520 & 0.5660 & 7.5351 & 5.3129 & 1.6579 & 0.436 \\
\hline 20 & 0.590 & 0.5698 & 10.6701 & 7.5169 & 1.6761 & 0.460 \\
\hline 25 & 0.670 & 0.5450 & 14.3689 & 10.8414 & 1.5048 & 0.494 \\
\hline 30 & 0.740 & 0.4817 & 22.9466 & 14.9828 & 0.8909 & 0.529 \\
\hline 40 & 0.850 & 0.3546 & 33.9584 & 25.0997 & -1.2622 & 0.596 \\
\hline 50 & 0.920 & 0.4573 & 41.1720 & 31.5652 & 1.1310 & 0.654 \\
\hline 60 & 1.000 & 0.1027 & 74.7257 & 54.4119 & -12.1089 & 0.705 \\
\hline 80 & 1.030 & 0.0862 & 96.6673 & 75.8608 & -13.2440 & 0.783 \\
\hline 100 & 1.050 & -0.1322 & 141.7377 & 120.9956 & -37.5723 & 0.834 \\
\hline 120 & 1.025 & -0.0341 & 168.1333 & 127.3424 & -23.1770 & 0.868 \\
\hline 160 & 1.015 & -0.0221 & 206.5651 & 166.0702 & -21.1139 & 0.906 \\
\hline 200 & 1.010 & -0.0446 & 261.6705 & 211.4298 & -25.9786 & 0.927 \\
\hline
\end{tabular}

$\left(^{*}\right)$ Lisempio numerico di trasformazione di una curva teorica di resistività differenziale trasversa nella corrispondente curva di schlumberger. Le colonne riportano i valori di $\varrho_{f_{1}}\left(l_{j}\right)$ in corrispondenza di prefissati valori $l_{j}$ dolla spariatura $L$; quindi i dati di $b_{j}, \beta_{j}, \gamma_{j}, c_{j}$ e $\bar{g}_{s}\left(l_{j}\right)$ ot tenuti rispettivamente dalle relazioni [3.11]. [3.16], [3.15]. [3.14] e [3.9]. 
I dati di $\bar{\varrho}_{s}\left(l_{j}\right)$, ottenuti dalla trasformazione e riportati nella Tab. 1, sono visibili nella stessa fig. 2 congiuntamente alla curva teorica di Schlumberger, relativa allo stesso modello e prelevata da Orellana e Mooney (1).

Come si può notare, i punti trasformati si dispongono perfettamente su quest'ultima.
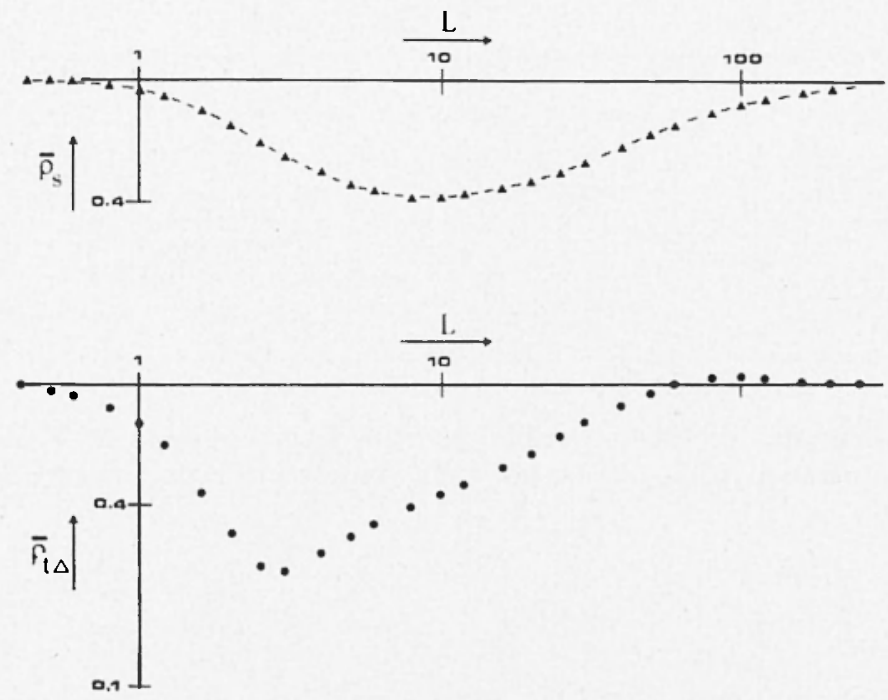

Fig. 2 - Esempio di trasformazione per una sezione di tipo $H$. (•) valori di $\underline{o}_{t, 1} ;(\Delta)$ valori di $\bar{\varrho}_{\varepsilon}$ ottenuti dalla trasformazione: $\left(--_{-}\right)$curva teorica di Schlumberger.

b) Sezione geoelettrica di tipo $K$

Isa fig. 3 mostra la trasformazione della curva teorica di $\varrho_{t, 1}$ per la situazione

$$
\varrho_{1}-1, \varrho_{2}=5, \varrho_{3}=1 ; h_{1}=1, h_{2}=25
$$

c) Sezione geoelettrica di tipo $A$

Isa fig. 4 mostra la trasformazione della curva teorica di $\varrho_{t / 1}$ per la situazione

$$
\varrho_{1}=1, \varrho^{0}=5, \varrho_{3}=20 ; h_{1}=1, h_{2}=25 .
$$



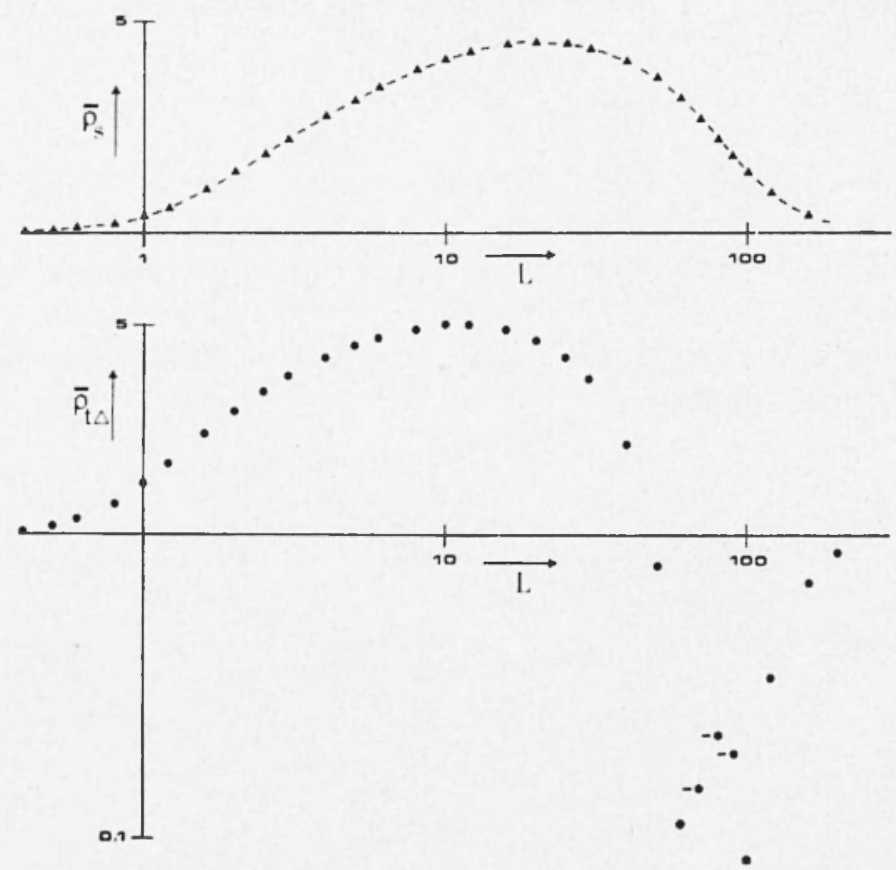

Fig. 3 - Esempio di trasformazione per una sezione di tipo $h$. Per il significato dei simboli v. fig. 2 . In più $(-\bullet)$ rappresenta valori negativi di $\varrho_{t, 1}$.
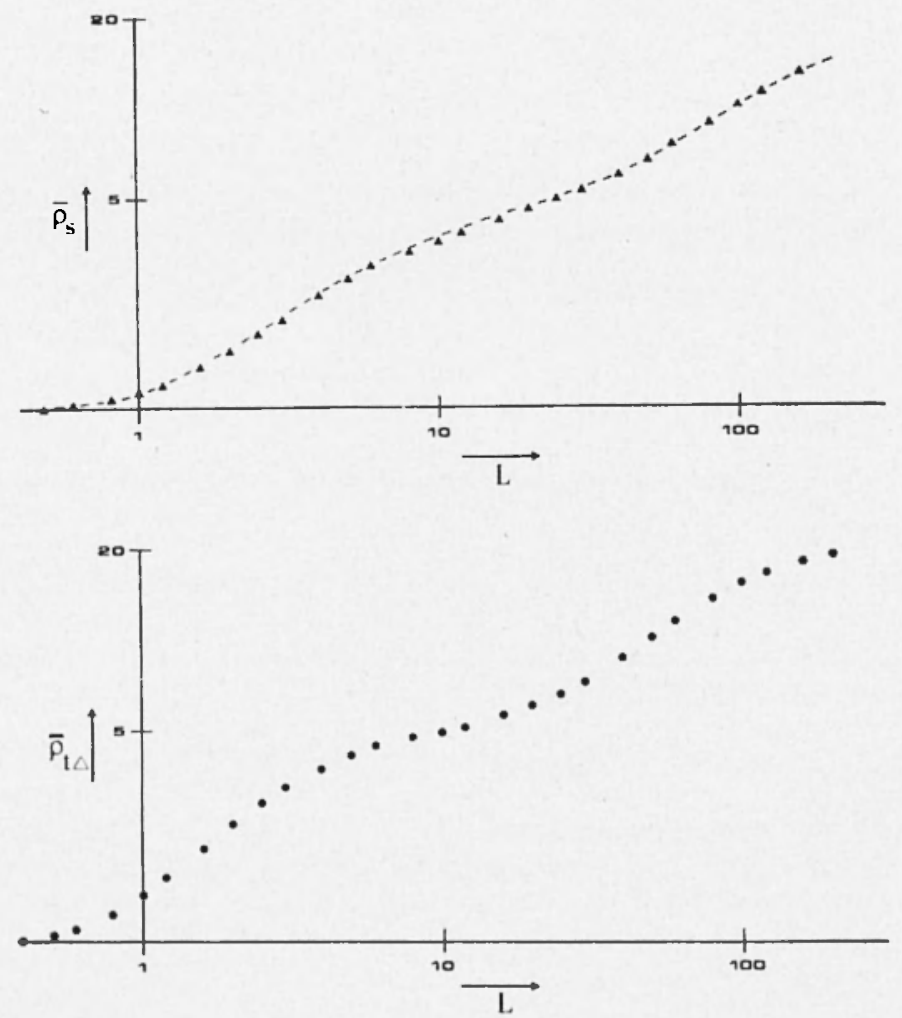

Fig. 4 - Esempio di trasformazione per una sezione di tipo $A$. Per il significato dei simboli v. fig. 2 . 
d) Sezione geoelettrica di tipo Q

Infine la fig. 5 mostra la trasformazione della curva teorica di $\bar{\varrho}_{t \Delta}$ con parametri

$$
\varrho_{1}=1, \varrho_{2}=0.4, \varrho_{3}=0.2 ; h_{1}=1, h_{2}=15 .
$$
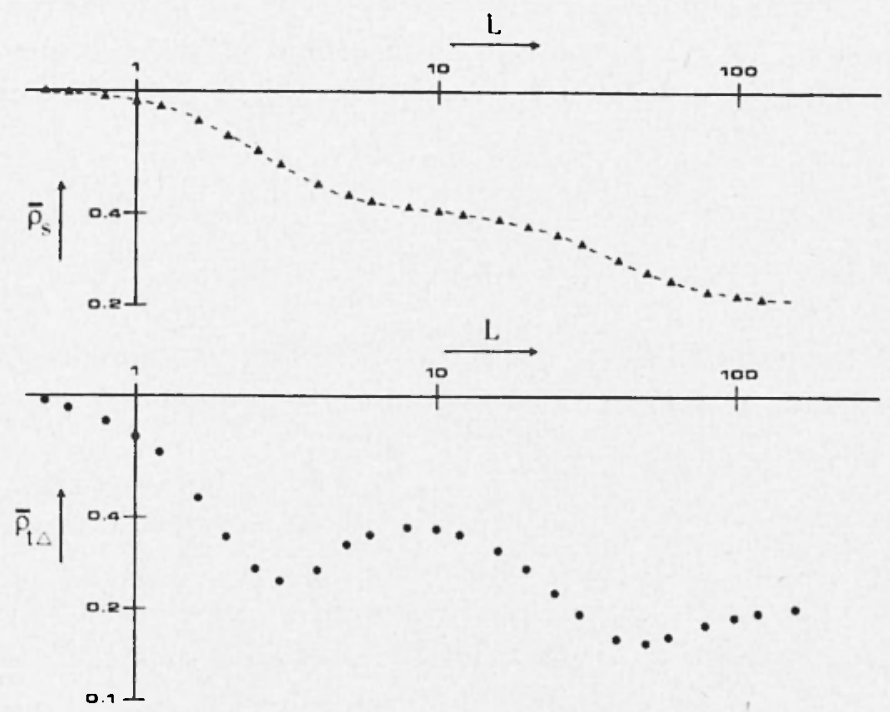

Fig. 5 - Esempio di trasformazione per una sezione di tipo $Q$. Per il significato dei simboli $\nabla$. fig. 2.

\section{6. - Conclusioni}

a) Da quanto si è detto, risulta che la trasformazione di una curva di $\bar{o}_{i, i}$. nella forma che si sarebbe ottenuta con il dispositivo di Schlumberger, è sempre possibile purché si faccia una determinazione accurata della resistività vera dello strato superficiale.

Per il suo elevato potere risolutivo, un sondaggio di resistivitì differenziale trasverso dà la possibilità di ottenere una buona ed esauriente interpretazione qualitativa della struttura del sottosuolo. La susseguente trasformazione in curva di Schlumberger permette di compiere, senza dover costruire apposite curve teoriche di $\bar{Q}_{t_{1}}$, quella interpretazione quantitativa che meglio è in accordo con la precedente analisi qualitativa. 
b) Rispetto al dispositivo di Schlumberger, quello differenziale ha lo svantaggio $\left(^{4}\right)$ di richiedere una maggiore energia, a parità di stendimento, per ottenere un valore misurabile di $\Delta(\Delta V)$. Ciò può provocare serie difficolti durante un intero sondaggio. Se, comunque, la necessità di ottenere informazioni dettagliate è ristretta a strutture non molto profonde, questo metodo di trasformazione permette di iniziare un sondaggio col dispositivo differenziale e di finirlo con lo Schlumberger. La trasformazione della prima parte del grafico ì ancora possibile con il risultato di ottenere un'unica curva di Schlumberger.

\section{APPENDICE}

Se il rapporto $L_{2} / L_{1}$ (v. fig. 1) è sufficientemente prossimo ad uno, allora le relazioni [1.1] e [1.2] possono essere approssimate come segue

$$
\begin{gathered}
\bar{r}_{L A}=\frac{1}{\lim _{\Delta I \rightarrow 0} \frac{\Delta\left(L / \bar{\varrho}_{s}\right)}{\Delta L}} \simeq \frac{L_{2}-L_{1}}{L_{2} / \bar{\varrho}_{s 2}-L_{1} / \bar{\varrho}_{s 1}} \\
\vec{o}_{i \Delta}=\lim _{\Delta L \rightarrow 0} \Delta\left(\bar{\varrho}_{s} L\right) / \Delta L \simeq\left(\varrho_{s 2} L_{2}-\bar{\varrho}_{s 1} L_{1}\right) /\left(L_{2}-L_{1}\right)
\end{gathered}
$$

dove $\bar{\varrho}_{\triangleleft 1}$ e $\bar{\varrho}_{s 2}$ sono le resistività apparenti Schlumberger per le configurazioni $A M N B$ e $B^{\prime} M N \Lambda^{\prime}$ rispettivamente (v. fig. 1) e date da

$$
\begin{aligned}
& \bar{o}_{s 1}=K_{1} \Delta V_{1} / I_{1} \\
& \bar{o}_{s 2}=K_{2} \Delta V_{2} / I_{2}
\end{aligned}
$$

Sostituendo le $[A .3]$ e $[A .4]$ nelle $[\Lambda .1]$ e $[\Lambda .2]$ si ottiene

$$
\begin{aligned}
& \bar{\varrho}_{L \Delta} \simeq \frac{K_{1}\left(L_{2}-L_{1}\right)}{L_{1} I_{1}} \frac{\Delta V_{1} \Delta V_{2}}{\frac{I_{2}}{I_{1}} \frac{L_{2} K_{1}}{L_{1} K_{2}} \Delta V_{1}-\Delta V_{2}} \\
& \bar{\varrho}_{t \Delta} \simeq \frac{K_{1} L_{1}}{\left(L_{2}-L_{1}\right) I_{1}}\left(\frac{K_{2} L_{2}}{K_{1} L_{1}} \frac{I_{1}}{I_{2}} \Delta V_{2}-\Delta V_{1}\right)
\end{aligned}
$$

Quando il rapporto delle correnti $I_{1}$ e $I_{2}$ è scelto in maniera tale che per l'LDR sia

$$
I_{1} / I_{2}=L_{2} K_{1} / L_{1} K_{2}
$$


e per il TNR

$$
I_{1} / I_{2}=L_{1} K_{1} / L_{2} K_{2}
$$

le $[\Lambda .5]$ e $[\Lambda .6]$ diventano

$$
\begin{gathered}
\bar{\varrho}_{L J 1} \simeq \frac{K_{1}\left(L_{2}-L_{1}\right)}{L_{1}} \frac{1}{I_{1}} \frac{\Delta V_{1} \Delta V_{2}}{\Delta T_{1}-\Delta V_{2}} \\
\bar{\varrho}_{\ell A 1} \simeq \frac{K_{1} L_{1}}{L_{2}-L_{1}} \frac{1}{I_{1}} \cdot\left(\Delta V_{2}-\Delta V_{1}\right)
\end{gathered}
$$

Ponendo, infine,

$$
\begin{aligned}
& K_{L}=K_{1}\left(L_{2}-L_{1}\right) / L_{1} \\
& K_{\ell}=K_{1} L_{1} /\left(L_{2}-L_{1}\right)
\end{aligned}
$$

si ottengono dalle [A.9] e [A.10] le [1.3] e [1.4] riportate precedentemente.

\section{BIBLIOGRAFIA}

(1) ORELAsi E., Moosey II. M., 1966. - Master lables and curves for verlical electrical sounding over layered structures. Madrid, Interciencia, 150 pp. and 66 tahles.

(-) Patrua I)., 1973. - On the transformation of dipole to Schlumberger sounding curces. Paper presented at the thirty-fifth Meeting of the European Association of Exploration Geophysicists, held at Brighton, 5th-8th .June, 1973 .

( $\left.{ }^{3}\right)$ RaBinovicir B. I.. 1965. - Fundamentals of the method of field difierence. "Priklatnaya Geofizika ", 43, pp. 47-59.

(4) ZoIIJY A. A. R., 1969. - A new melhod for differential resistivity sounding. "Geophysics", 34, pp. 924-43. 